\title{
The Adrenal Gland: Common Disease States and Suspected New Applications
}

\section{LINDA S. GORMAN}

\section{LEARNING OBJECTIVES}

1. Explain the renin-angiotensin-aldosterone mechanism and how it maintains blood pressure.

2. Describe the present laboratory findings for a soldier suffering from Post Traumatic Stress Disorder (PTSD).

3. Characterize how we differentiate between the various forms of Cushing's syndrome and the testing that makes that possible.

4 Describe the symptoms and laboratory findings for a patient with Addison's disease.

5 Characterize how we deduce a finding of pheochromocytoma in a patient.

ABBREVIATIONS: ACTH - adrenocorticotropic hormone; ACE - angiotensin converting enzyme; ARR aldosterone-renin ratio; $\mathrm{CAH}$ - congenital adrenal hyperplasia; $\mathrm{CRH}$ - corticotropin releasing hormone; CT - computerized axial tomography; DHEA - dehydroepiandrosterone; 11-DOC - 11-deoxycorticosterone; GR - glucocorticoid receptor; HPLC-high pressure liquid chromatography; HIV - human immunodeficiency virus; LC/MS-MS - liquid chromategraphy/mass spectroscopy-mass spectrophotometry; MRI - magnetic resonance imaging; MS - mass spectroscopy; PTSD - post-traumatic stress disorder; RIA - radio-immunoassay; TB - tuberculosis; SRE steroid response element; VMA - vanillylmandelic acid

INDEX TERMS: Adrenal Gland, Adrenal Hyperplasia, Adrenocortical Adenoma, Aldosterone, Hyperaldosteronism, Hypokalemia, Renin, Adrenal Insufficiency

Clin Lab Sci 2013;26(2):118

Linda S. Gorman, PhD, MLS (ASCP) ${ }^{C M}$, University of Kentucky, Lexington, $K Y$

Address for Correspondence: Linda S. Gorman, PhD, $M L S(A S C P)^{C M}$, CLS Education Co-ordinator, Associate Professor, 900 S. Limestone Ave, Rm 126G CTW,
University of Kentucky, Lexington, KY 40536-0200, (859)-218-0855,lsgorm0@uky.edu

This article covers the physiology of the adrenal gland as well as the common disease states. Post-traumatic stress disorder is discussed in relation to the possible role of the adrenal gland hormones and this disorder. Laboratory testing used to diagnose adrenal disorders is briefly described using reference laboratory methods.

Historically, the adrenal gland was thought to be "excess renal" tissue and not significant by anatomist Batholemeus Eustachius (1520-1574). ${ }^{1}$ In the early 1800 's, Culver (1769-1832) was the first to recognize the difference between the outer layer of the adrenal gland and the inner central core. It would not be until 1836 before N. Nagel termed these as "cortical" for the outer layer and "medulla" for the inner layer. Definitive descriptions of the adrenal gland awaited the microscopic anatomical examination by R. A. Von Kolliker (1817-1905). He described his findings as "the cortical and medullary substances are physiologically distinct and have different functions."

The adrenal gland is viewed as a pyramid-shaped gland that is positioned above each kidney within the human body (Figure 1). This gland can be divided into the cortex and medulla regions, with the cortex producing steroid type hormones and the medulla producing neuropeptide type hormones. Embryonic development of the cortex comes from mesothelium adjacent to the dorsal mesentery. ${ }^{2}$ The adrenal cortex differentiates into 3 zones- the glomerulosa, fasiculata and reticularis. Development of the adrenal cortex leads to the zona glomerulosa and zona fasiculata being present at birth with the zona reticularis presence delayed until the $3 \mathrm{rd}$ year after birth. The embryonic development of the adrenal medulla comes from the neural crest cells that migrate to a cavity of the developing cortex. Initially these neuron-like cells are not encapsulated by the fetal cortex. During month two of development these cells 
are encapsulated and the cell types that develop are $80 \%$ epinephrine-secreting and $20 \%$ norepinephrinesecreting in the adult. ${ }^{2}$ The mature adrenal gland is $90 \%$ adrenal cortex and $10 \%$ adrenal medulla. ${ }^{3}$

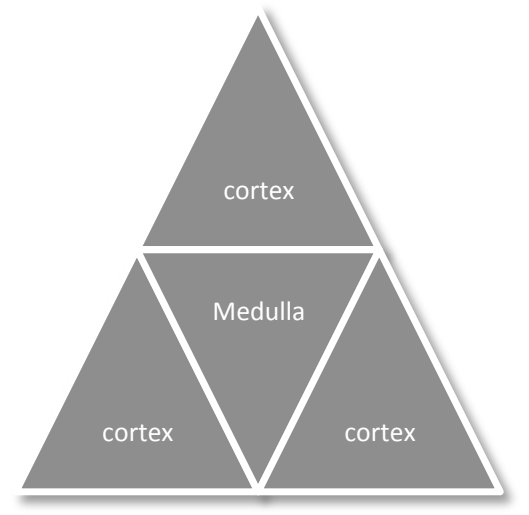

Figure 1.Adrenal gland with cortex and medulla

The adrenal cortex, with its 3 distinct zones, produces steroid type hormones from the common substrate pregnenolone, formed in the cortex biochemical pathway from cholesterol to make various steroid hormones unique to the adrenal gland. The outer layer of the cortex is called the zona glomerulosa. Production of the steroid hormone aldosterone in this zone leads to regulation of body sodium levels and influences blood pressure by altering fluid volumes. This layer is a regulator of salt content in blood and fluids. ${ }^{3}$ Aldosterone plays a key role in regulating the kidney retention of sodium as well as potassium release and thus regulation of blood-fluid volumes. The zona fasiculata is known for its use of the common substrate pregnenolone to make cortisol and other glucocorticoids of interest. Cortisol is the major hormone of interest and influences glucose homeostasis, protein catabolism, and blood pressure to some extent. ${ }^{4}$ The zona reticularis produces the androgens made by the adrenal gland, in particular DHEA or dihydroepiandrosterone and the sulfated form DHEAS. ${ }^{4}$

\section{Aldosterone}

Aldosterone's physiologic impact is to regulate sodium's affect on blood-fluid volume in the circulation. Classically, aldosterone regulates the reabsorption of sodium from the effulate leaving the kidney nephron for the collecting duct. At the distal nephron or the receptors in the renal cortical collecting ducts of the kidney, the presence of aldosterone facilitates the reabsorption of sodium into the bloodstream, the passive ejection of potassium to the collecting duct, and the increase in blood pressure of circulation leaving the kidney. ${ }^{6,7}$ Electrolytic regulation exerted by aldosterone has a unique feedback mechanism for regulating aldosterone secretions. The renin-angiotensin system is described in Figure 2. Renin is a proteolytic enzyme released from the juxtaglomerular apparatus of the kidney when the blood flow or blood pressure through the renal artery is low. Renin release enzymatically acts on angiotensinogen from the liver to form angiotensinI. Angiotensin-I traveling in the bloodstream is enzymatically changed to angiotensin-II by the lung enzyme angiotensin converting enzyme (ACE). Angiotensin-II is a potent vasoconstrictor that constricts blood vessel muscle to increase blood pressure as it stimulates the adrenal gland to release aldosterone from the adrenal cortex zona glomerulosa. This increase in aldosterone secretion will foster sodium reabsorption from the distal nephrons of the kidney. ${ }^{6}$ As the sodium content increases in the bloodstream it changes the salt content and raises the blood pressure. An increased blood pressure then shuts off the renin release causing the renin-angiotensin-aldosterone system to shutdown until the blood pressure thru the kidney is low again.

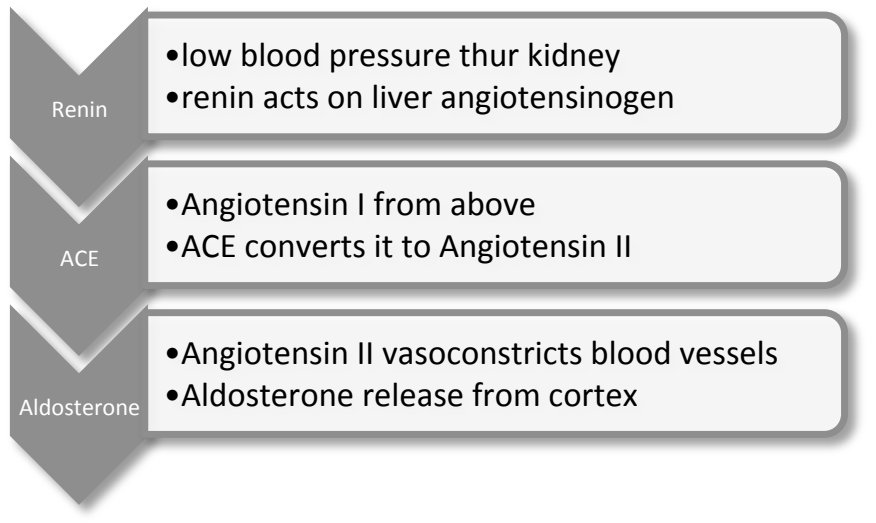

Figure 2. Renin-Angiotensinogen-Aldosterone mechanism.

The common precursor to aldosterone and cortisol production is the conversion of cholesterol to pregnenolone, a common steroid precursor. Within the glomerulosa layer where aldosterone is formed there is an aldosterone synthetase enzyme that fosters the production of this hormone from pregnenolone precursor products. ${ }^{3}$ The presence of this enzyme in other cortex layers is circumvented by the activity for aldosterone synthetase being low in the fasciculata and the reticularis layers. Along the same lines the enzyme 17-alpha-hydroxylase, which is important in the 
production of cortisol and its precursors from pregnenolone, has a low activity rate in the glomerulosa layer, but active in the fasiculata layer. ${ }^{3}$ More aldosterone is generated by the renin-angiotensinaldosterone system than by the negative feedback mechanism used for cortisol production. ${ }^{3}$ This divergence in controlling pathways for the two major adrenal hormones contributes to their ability to work in concert or individually.

\section{Cortisol}

Cortisol release from the adrenal cortex results from the hypothalamus stimulation of the pituitary to release ACTH. ACTH acts on the adrenal cortex to foster cortisol release. Cortisol travels in the blood to its target of interest attached to Cortisol-binding globulin (CBG). Cortisol attaches at the cellular level to a glucocorticoid receptor (GR) and enters the cell. ${ }^{5}$ This cortisol-GR complex moves thru the cell cytoplasm to the cell nucleus, where it will affect responsive genes or the steroid-binding element (SBE). The messenger RNA (mRNA) generated by the cortisol action on the nucleus leads to the formation of proteins/enzymes that will influence the cell's function or metabolism. Cortisol from the adrenal gland also acts as the negative feedback compound to the hypothalamus (CRH) and pituitary (ACTH) leading to suspension of their respective hormonal actions. Cortisol expresses diurnal variation with high blood concentrations in the morning and low concentrations at midnight. Morning reference ranges typically are $7-25 \mathrm{ug} / \mathrm{dL}$ while evening ranges run 2-9 ug/dL with midnight cortisol levels less than $5 \mathrm{ug} / \mathrm{dL} .{ }^{5,9}$

Cortisol, from the zona fasiculata, influences glucose levels, protein function of those proteins involved in inflammation, circulating levels of free fatty acids, and body response to stress. ${ }^{5,6}$ Cortisol helps to maintain normal glucose levels by increasing the gluconeogenic process when blood glucose levels fall. Cortisol induces lipid breakdown and amino acid release from muscle so that these gluconeogenic substrates can be turned into glucose and stored as liver glycogen. ${ }^{3}$ Winter et. al. report that this gluconeogenic process of cortisol can lead to insulin resistance in skeletal muscle, liver and adipose tissue. ${ }^{5}$ They also state that excess cortisol can produce diabetes mellitus. The lipid lipolysis that cortisol fosters in adipose tissue increases the level of circulating free fatty acids. Winter et. al. state that excess cortisol affecting lipids can thus lead to hypertriglyceridemia and/or hypercholesterolemia, indicating the patient can develop risk for lipid associated pathologies. ${ }^{5}$ As for the protein effect of cortisol, its anti-inflammatory effect makes cortisolderived drugs useful in treating diseases like arthritis, inflammation, and dermatitis. ${ }^{5}$ Normal body immune function uses the cortisol affect on proteins to suppress immune response to inflammation and the allergic response. ${ }^{6}$ The influence of cortisol on the body's response to stress is seen in how the cortisol hormone in high doses leads to stronger heart contractions, increased heart rate, and blood vessel tone with decreased endothelial permeability. ${ }^{5}$ These actions help maintain body readiness to move. Cortisol also affects appetite, wakefulness, mood, and behavior.

Cortisol changes associated with stress have been examined in soldiers with post-traumatic stress disorder (PTSD). ${ }^{8}$ PTSD can lead to soldier-patient depression, insomnia, swings in mood from happy to anger and rage with little provocation. Medical teams have tried to find physiological and psychological reasons for this condition. In the laboratory the work-up for PTSD will show decreased cortisol levels with increases in norepinephrine and epinephrine levels. ${ }^{8}$ The hypothalamic-pituitary-adrenal negative feedback mechanism can be abnormal in its response to the stress of PTSD. Morning cortisol levels are lower than the typical 7-25 $\mathrm{ug} / \mathrm{dL}$. This finding is not fully validated yet and may be related to the upswing in natural opiates produced in these soldiers when faced with danger or the perceived danger of the past. These natural opiates may cause the affected soldier to be uninvolved with family, friends, and healthcare providers. Whether the cortisol and catecholamine changes are the cause or result of PTSD is yet to be determined.

Medications for soldiers with PTSD have been used to address the psychosis, sleep issues, and rage/anxiety flare-ups. Medications directed at specific symptoms like benzodiazepines for anxiety, anti-depressants for depression and clonidine for nightmares are being prescribed. ${ }^{8}$ New studies using alpha-1-antagonists have improved the sleep-associated symptoms of PTSD and low-dose glucocorticoids (cortisol) seem to decrease the trauma of past events of war in soldiers afflicted with PTSD. ${ }^{8}$ 


\section{Cortisol Measurements}

Cortisol measurements on specimens of plasma, serum, or urine typically use immunoassays where the tag or marker for cortisol is attached to the antibody. ${ }^{9}$ Reference laboratory methods for cortisol use a chemiluminescent immunoassay where the label is released from a substrate affording a strong signal for detection. Results from immunoassays are tempered by the realization that a number of steroid-like compounds in the cortisol synthetic pathway can crossreact with the antibody used in these immunoassays. ${ }^{5}$ Winter et. al. lists the drug prednisolone as having a $171 \%$ crossreactivity with cortisol in such assays. ${ }^{5}$ Thus a patient on cortisol therapy would not give a reliable answer for cortisol using these types of immunoassay methods. Dexamethasone has a very low cross-reactive capacity $(<0.08 \%)$ with immunoassays so its utility in suppression testing is not compromised. ${ }^{5}$ At this time, the superior method for performing cortisol assays is to use the mass spectrophotometric (MS) method. According to Winter et.al. the MS method is the best for tracking free cortisol levels. ${ }^{5}$ Laboratories without mass spectroscopy capabilities can perform the immunoassay but should follow up by sending the specimen to a reference laboratory for mass spectrophotometer assay. Kit methods for urinary free cortisol may require sample pre-testing preparation before performing the assay. Other immunoassay kits exist for the measurement of ACTH, but specimen integrity is an issue and must be strictly adhered to when this assay is needed. CRH is usually not measured in cases of suspected cortisol deficiency or excess. ${ }^{5}$

\section{Aldosterone measurements}

Measurement of aldosterone varies from radioimmunoassay (RIA) to fluorimetric immunoassays. Since this is not a commonly performed laboratory procedure, reference laboratories perform the majority of aldosterone assays. Quantitative RIA seems to be the dominate methodology used by reference facilities to measure aldosterone. ${ }^{10}$ Serum and urine samples have to be collected under controlled conditions to provide the best results. The dietary sodium level should be between $100-200 \mathrm{mEq} /$ day for approximately 3 days before the collection of urine and/or serum for this assay. ${ }^{10}$ The reference range for serum aldosterone of an upright patient draw is $4.0-31.0 \mathrm{ng} / \mathrm{dL}$ using a quantitative radioimmunoassay. ${ }^{11}$ Urine aldosterone levels are obtained from a 24-hr collection that has been preserved with 1 gram of boric acid per $100 \mathrm{~mL}$ of urine or has had the $\mathrm{pH}$ adjusted with $6 \mathrm{M} \mathrm{HCl}$ or $50 \%$ acetic acid to $\mathrm{pH} 2-4$.

Aldosterone/renin ratios (ARR)s are used to determine hyperaldosteronism. ${ }^{12}$ Measurements of aldosterone and renin from properly collected specimens, when the patient is supine or upright for 2 hours, are used to calculate the ARR. If the ratio is greater than 25, with an aldosterone value of $15 \mathrm{ng} / \mathrm{dL}$ or more, the patient is believed to have primary hyperaldosteronism. ${ }^{12}$ The ratio in secondary hyperaldosteronism is less than 25 as is the ARR for a patient with Addison's disease.

\section{Adrenal Diseases}

Classically when discussing the disease states of the adrenal gland, we divide them into cortex disorders and medulla disorders. The adrenal cortex, where aldosterone and cortisol are the major hormones, can be discussed as hyperadrenal diseases or hypoadrenal diseases. The hormone aldosterone can be classified as either a hypoaldosteronism state or hyperaldosteronism state when the adrenal disease affects only the outer adrenal cortex. Genetic disorders such as congenital adrenal hyperplasia (CAH), affecting a small group of patients, are not included in this discussion of adrenal disorders. For cortisol the diseases of interest are Addison's disease (hypocorticolism) or Cushing's syndrome (hypercorticolism). For the adrenal medulla pheochromocytoma, due to increased epinephrine levels, is the primary disorder of interest with no significant disorder associated with low medulla activity. The interplay between the various layers of the adrenal cortex and the fact that negative feedback mechanisms regulate these hormones means that most of the adrenal disorders discussed here can show an effect on other adrenal hormones. The primary hormone marker for each of these diseases stands out but other adrenal hormones can show variation from reference range levels when the disease is present.

\section{Hypercorticolism or Cushing's Syndrome}

Excesses of cortisol are seen in Cushing's syndrome. Cushing's syndrome is rare, with $90 \%$ occurring in adulthood, and an incidence of 2 new cases per million population per year. ${ }^{13}$ While an adrenal tumor in the adrenal cortex would be a primary hypercorticolism and source for Cushing's syndrome, the majority of cases of Cushing's are due to exogenous cortisol administra- 
tion. ${ }^{3,5,6}$ Non-exogenous incidents of Cushing's can be divided into ACTH-independent and ACTHdependent Cushing's syndromes. In adults, the ACTHindependent Cushing's is only $10-20 \%$ of the Cushing cases seen. These patients have a tumor in their adrenal gland that often needs to be removed due to the risk of malignancy associated with such neoplasms. ${ }^{13}$ The adult patients with ACTH-dependent Cushing's constitute $80-90 \%$ of the Cushing's cases and often have a pituitary tumor that is secreting ACTH in excess of patient needs. ${ }^{13}$

Cushing's syndrome patients exhibit a number of physical symptoms that can initiate laboratory testing and screening for the disease. The patient's physical appearance can include weight gain with much of the weight located centrally around the abdomen. Patients often have a classic "moon face" or rounding to their facial features, along with the "buffalo hump" or fat deposition across the back and shoulders. Adult patients complain about poor wound healing, collapsing spinal discs, cataracts, hirsutism, acne, and hypertension. Often the patient has a voracious appetite and increased susceptibility to infections. ${ }^{3,13}$ Given cortisol functions, the excess cortisol in Cushing's syndrome can lead to compromise of the patients' immune function, loss of bone development leading to osteoporosis, renal hypertension, and metabolic issues from insulin resistance. $^{3}$

Laboratory testing (Figure 3) to confirm Cushing's syndrome includes testing for cortisol as well as complete metabolic profile. Decreases in potassium and increases in total carbon dioxide follow with elevated glucose values and a possible decrease in patient eosinophil count. ${ }^{13}$ Cortisol is measured on samples taken at $8 \mathrm{am}$ and $4 \mathrm{pm}^{7}$ Diurnal variation of cortisol secretion necessitates the timed draws. Cortisol values at $8 \mathrm{am}$ are normally between $7-25 \mathrm{ug} / \mathrm{dL}$ and $4 \mathrm{pm}$ cortisol levels are 2-9ug/dL, demonstrating the expected variation in blood levels. ${ }^{13}$ Patients with Cushing's syndrome lose this diurnal variation and their evening cortisol value is often $>15 \mathrm{ug} / \mathrm{dL}^{3}$. The difficulty in differentiating Cushing's syndrome as either ACTHindependent or ACTH-dependent types is compounded by the fact that both demonstrate this loss of cortisol diurnal variation.

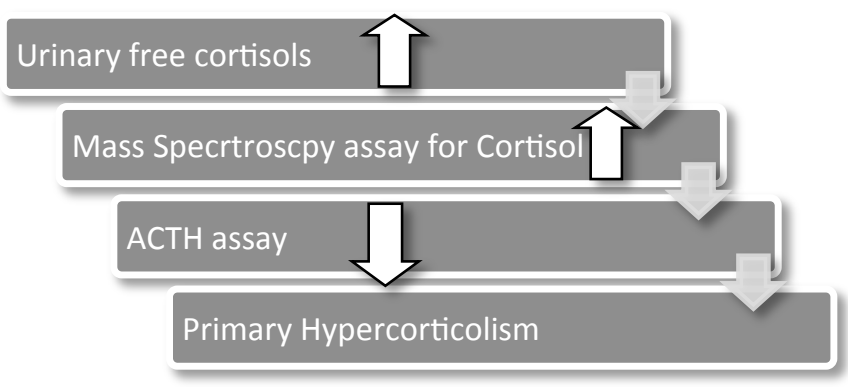

Figure 3. Laboratory testing for Cushing's syndrome, Primary hypercorticolism

By including ACTH assays, further differentiation can occur. The immunoassay for ACTH is often a quantitative chemiluminescent immunoassay with a range of $6-69 \mathrm{pg} / \mathrm{mL}^{14}$ When the patient's evening cortisol level is $>15 \mathrm{ug} / \mathrm{dL}$ and the ACTH level is $<5$ $\mathrm{pg} / \mathrm{mL}$, the problem is believed to be a tumor in the adrenal gland and thus an ACTH-independent issue. When the ACTH level is greater than its upper range, as is the cortisol, the problem is said to be an ACTHdependent Cushing's pathology. ${ }^{3}$ While this assay is useful it is often not practical for local laboratories. Better screening for suspected Cushing's syndrome is achieved using the 24-hour urine collection and assaying for free cortisol, the high-dose dexamethazone test, and/or the salivary collections for cortisol and ACTH collected at midnight. ${ }^{3,5}$ The urinary free cortisol level should be determined by tandem mass spectroscopy which has a $95-100 \%$ sensitivity and $98 \%$ specificity. ${ }^{3}$ One should use creatinine determinations to verify the 24-hour collection as random urine collections do not give a true representation of the cortisol cyclic secretions. ${ }^{5}$

High dose dexamethazone testing is used to differentiate ectopic ACTH-secreting tumors from pituitary ACTHsecreting tumors. Patients are given 8-12 $\mathrm{mg}$ of dexamethazone at $11 \mathrm{pm}$. The next morning blood collections for ACTH and cortisol plus urine for free cortisol concentrations are collected. ${ }^{3}$ The suppression of ACTH, cortisol and urinary free cortisol concentrations after dexamethazone high-dose indicates a pituitary ACTH-dependent tumor. Lack of suppression after this dexamethazone suppression test indicates an ectopic ACTH-secreting tumor. Salivary sampling is useful if the patient must be sampled at midnight or have frequent sampling as with proving a case of intermittent Cushing's syndrome. ${ }^{3}$ 


\section{FOCUS: ENDOCRINOLOGY}

When Cushing's syndrome is due to ACTHindependent causes, the aldosterone level tends to be regulated by the renin-angiotensin-aldosterone process because the ACTH level is low and not stimulating the adrenal cortex. In Cushing's syndrome due to ACTHdependent causes, there is an increase in stimulation of the adrenal cortex to produce more aldosterone as well as more cortisol. While there may be slight increases in aldosterone level under these circumstances, the renin role helps to minimize the change in aldosterone.

Once the laboratory data confirms the high probability of Cushing's syndrome, the patient will undergo a MRI or CT scan that will locate the cortisol tumor in the adrenal gland or if an ACTH-dependent tumor, located in the pituitary, lung, or GI tract. ${ }^{3,13}$ Ectopic ACTH secreting tumors are usually malignant making up 15\% of the causes of Cushing's Syndrome. ${ }^{3}$ Pituitary ACTHsecreting tumors constitute $68 \%$ of the cause for Cushing's syndrome while adrenal tumors constitute $17 \%$ of the cause. ${ }^{3}$ Verification by 2 laboratory screening tests for elevated cortisol are required before an MRI or CT scan can be used to identify the tumor location. Surgery is the best option, but radiation and/or medication to suppress the adrenal cortisol production can also be used. ${ }^{3}$ Should surgery lead to adrenal removal, the cortex hormones aldosterone and cortisol need daily replacement via medication. ${ }^{3}$

\section{Hypocortisolism or Addison's Disease}

A deficiency of cortisol from the adrenal cortex fasciculata layer leads a patient to complain about weakness, fatigue, weight loss, and anorexia in $100 \%$ of the cases. Nausea and diarrhea complaints occur in 50\% of the cases, and pain in only $10 \%$ of the cases. ${ }^{3}$ Patients with Addison's disease often exhibit hyperpigmentation and adrenal gland calcification. ${ }^{3}$ Because the entire adrenal cortex is affected, this condition exhibits hypotension and glucose deficiency abnormalities. It is not unusual for a patient with Addison's disease to have both a low cortisol level and aldosterone level. The lack of cortisol means less fatty acid and amino acid release and therefore less gluconeogenesis resulting in lower blood glucose levels during fasting states. ${ }^{3,5}$ The lack of aldosterone results in decreased sodium reabsorption by the kidneys and a decrease in blood pressure, resulting in hypotension. The laboratory assessment of a patient with these symptoms will confirm the patient's Addison's disease pathology.
Laboratory markers for Addison's disease include cortisol, electrolytes and glucose. The electrolytic picture shows a decreased sodium value, increased potassium level, and slightly decreased total carbon dioxide ( $\mathrm{tCO} 2$ ), indicating a mild metabolic acidosis. Additionally the serum total calcium level will be increased and the BUN may be elevated (pre-renal azotemia). ${ }^{3}$ Because the cortisol level is decreased, the fasting glucose value is also decreased, explaining the symptoms of weakness and fatigue. Morning cortisol levels will be low, while ACTH levels from samples at the same time will be $>200 \mathrm{pg} / \mathrm{mL}^{3}$ To further substantiate the cortisol findings, the patient can be given 250 ug dose of cosyntropin, a synthetic cortisol and aldosterone stimulator, after an overnight fast. ${ }^{5}$ The patient has a baseline blood draw at $8 \mathrm{am}$, then the drug, then a 30 minute and 60 minute blood draw. ${ }^{5}$ The patient with Addison's disease will have cortisol level $<20 \mathrm{ug} / \mathrm{dL}$ indicating a lack of adrenal function with no rise in cortisol seen following the drug stimulation. This testing sequence is capable of detecting primary adrenal insufficiency or hypocorticolism but not the secondary causes of Addison's disease.

Addison's disease or hypocortisolism is a pathology that is silently forming while the patient is unaware. Often over $70 \%$ of the adrenal gland has been lost before symptoms cause the patient to seek medical advice. Primary causes of adrenal insufficiency include autoimmune adrenal inflammation (70\%), infections (fungal, HIV, and TB), bilateral adrenal hemorrhage, infiltrative processes, and metastasis. ${ }^{7}$ Secondary causes of adrenal insufficiency are those that interfere with ACTH pituitary production, such as cortisol medications or pituitary tumors and various other abnormalities. $^{3}$

Differentiating primary adrenal insufficiency from secondary adrenal insufficiency can become frustrating due to a lack of clear answers. The cosynthopin stimulation test is not capable of differentiating between these conditions. Metyrapone will block the 11-Bhydroxylase enzyme and lead to an increase in 11deoxycortisol (11-DOC) with a decrease in cortisol production in normal patients. ${ }^{3}$ Giving metyrapone dose at midnight and seeing an abnormal response where the cortisol is not decreased can indicate a secondary cause for the adrenal insufficiency. ${ }^{3}$ MRIs can be used to detect brain and pituitary problems if there is 
no apparent cause for adrenal insufficiency, such as with exogenous cortisol treatments. The long term treatment of adrenal insufficiency is to replace the missing adrenal hormones. ${ }^{3}$

\section{Medullary disease}

Epinephrine formed by the adrenal medulla can be secreted into the blood circulation, allowing this hormone to act in seconds compared to the 20 minutes or so that cortisol action requires. ${ }^{3}$ The formation of epinephrine in the adrenal medulla chromaffin cells or pheochromoblasts begins with the amino acid phenylalanine and its conversion thru steps to tyrosine, then dopamine, thru norepinephrine, to epinephrine. The conversion of norepinephrine to epinephrine requires the enzyme phenylethanolamine $\mathrm{N}$-methyl transferase (PNMT), which is a cortisol-dependent enzyme. Epinephrine is housed in storage vesicles within the chromaffin cells of the medulla. These secretory storage vesicles allow for quick release of epinephrine into the blood circulation from the medulla when stimuli of stress or hypotension lead to its release from the medulla. ${ }^{3}$

Degradation of epinephrine produces the metanephrines and vanillylmandelic acid (VMA) products that can be measured to assess patient epinephrine output. While assays exist for measuring the epinephrine released from the medulla, the speed of degradation, uptake or removal of this hormone from the circulation makes successful assay completion very difficult. Epinephrine moves quickly from hormone action to removal as it moves from the blood to the target cell. The urinary catecholamines, namely free norepinphrine and epinephrine, can be measured by HPLC, fluorometric assays, or LC-MS/MS. ${ }^{3}$ Preserving the free catecholamines and metanephrines in urine enables the medical laboratory professional to determine the amount of epinephrine hormone released from the medulla. Elevations of the catecholamine content in a 24-hr urine followed by looking at the fraction that is metanephrine in origin, enables the medical laboratory professional to identify patients with pheochromocytomas.

Patients with hypertension symptoms and elevated metanephrine concentrations are evaluated for pheochromocytoma. This disease is a tumor of the chromaffin cells that leads to excessive release of epinephrine, resulting in serious hypertension and the accompanying jumpiness associated with excess epinephrine hormone being present. ${ }^{3}$ Surgery is the usual option to treat a medullary tumor. Replacement hormones of cortisol, aldosterone, and other adrenal products are necessary if the adrenal gland is removed in order to treat a pheochromocytoma.

\section{Summary}

The adrenal gland, while small in size, provides a major punch to human metabolism. The interplay between the adrenal cortex hormones aldosterone and cortisol provides needed regulation to human metabolism. Aldosterone regulates the body sodium content affecting blood pressure thru fluid-volume regulation by the kidney. Cortisol, also from the adrenal cortex, contributes to regulation of glucose and protein metabolism. Diseases like Addison's disease and Cushing's syndrome that affect the normal levels of these hormones can lead to serious pathologies that need to be detected thru clinical laboratory testing. The inner core of the adrenal gland, called the medulla, houses the catecholamine epinephrine, a fast acting neuropeptide hormone that can influence body action and energy levels quickly. The pheochromocytomas pathology of the adrenal medulla adversely affects the medulla hormones and needs to be recognized by clinical laboratory testing.

This overview of the adrenal gland and its potential pathologies needs to be looked at anew in relation to post-traumatic stress disorder to find any linkages that may aid in the treatment and cure of our affected military soldiers. This interrelationship between cortisol and epinephrine in PTSD should be closely evaluated to determine if the suspected linkages are significant.

\section{REFERENCES}

1. Leoutsakos, B. and Leoutsakos, A. "The adrenal glands: a brief historical perspective”, Homones 2008;7(4):334-6

2. Hill, M. 2012, UNSW Embryology ISBN: 9780733426094 - UNSW CRICOS Provider Code No. 00098G http://php. med.unsw.edu.au/embryology/index.php?title=Endocrine__Adrenal_Development\#Adrenal_Development. Access 2013 January 28

3. Hungerford, R and Meikle, A. "Endocrine Hormones", Chapter 20, Clinical Chemistry: Techniques, Principles, Correlations", 6th ed. In: Michael L. Bishop, Edward P. Fody, Larry E. Schoeff. Philadelphia: Lippincott, Williams, and Wilkins, (2010),458-74.

4. Kay, S. and Reynolds, M. Medscape Reference http:// emedicine.medscape.com/article/940347-overview\#aw2aab6b4 
Oct 22, 2008. Accessed 2013 January 12.

5. Winter, WE, Bazydlo, LAL, Harris, NS, "Cortisol Clinical Indications and Laboratory Testing" Clinical Laboratory News, 2012;38(9):8-10.

6. Sunheimer, R. and Graves, L. "The Endocrine Chapter" Upper Saddle River, New Jersey:Pearson Education , Inc, 2011.

7. Virk, R. "Clinical Chemistry: Adrenal Tests: Aldosterone", University of Massachusetts Memorial Hospital, revised Sept. 21, 2012, c. 2009-2010. http://www.pathologyoutlines.com/ topic/adrenalhyperaldosteronism.html. Accessed 2013 January 12.

8. Gore, T. and Ahmed, I. "Posttraumatic Stress Disorder Workup", Medscape Reference: Drugs, Diseases, Procedures, http://emedicine.medscape.com/article/288154-workup\#aw2 aab6b5b 2aa Accessed 2012 December 11.

9. ARUP Cortisol assay, ARUP Laboratory Test Directory: 0070030, http://www.aruplab.com/guides/ug/tests/0070030. jsp Accessed 2013 January 12.
10. ARUP Aldosterone urine assay, ARUP Laboratory Test Directory: 0070480, http://www.aruplab.com/guides/ug/tests/ 0070480.jsp Accessed 2013 January 12.

11. ARUP Aldosterone serum assay, ARUP Lab Test Directory: http://www.aruplab.com/guides/ug/tests/0070015.jsp Accessed 2013 January 11.

12. ARUP Aldos/renin ratio sheet, ARUP Lab Test Directory: 0070073, http://www.aruplab.com/guides/ug/tests/0070073. jsp Access 2013 January 12

13. Chrousos, G. and Kemp, S, et. al. "Glucocorticoid Therapy and Cushing's syndrome" Medscape Reference: Drugs, Diseases, Procedures, March 5, 2012. http://emedicine. medscape.com/article/921086-overview\#a0104 Accessed 2013 January 12 .

14. ARUP ACTH assay, ARUP Lab Test Directory: 0070010, http://www.aruplab.com/guides/ug/tests/0070010.jsp Accessed 2013 January 12.

\title{
CLEC 2014 Call for Abstracts
}

The deadline for abstracts for poster presentations or technology demonstrations at the 2014 ASCLS Clinical Laboratory Educators Conference (CLEC) is October 1, 2013.

\author{
Submission instructions and the proposal form may be found at \\ www.ascls.org/CLEC. The completed proposal form and abstract must be \\ submitted electronically by the deadline.
}

The 2014 CLEC will be held February 20-22 in San Jose, California. Additional meeting information will be available at www.ascls.org/CLEC.

The Focus section seeks to publish relevant and timely continuing education for clinical laboratory practitioners. Section editors, topics, and authors are selected in advance to cover current areas of interest in each discipline. Readers can obtain continuing education credit (CE) through P.A.C.E. ${ }^{\circledR}$ by completing the continuing education registration form, recording answers to the examination, and mailing a photocopy of it with the appropriate fee to the address designated on the form. Suggestions for future Focus topics and authors, and manuscripts appropriate for CE credit are encouraged. Direct all inquiries to the Clin Lab Sci Editorial Office, Westminster Publishers, 315 Westminster Court, Brandon MS 39047. (601) 214-5028, (202) 315-5843 (fax).westminsterpublishers@comcast.net. 